\title{
Highly efficient differentiation of human ES cells and iPS cells into mature pancreatic insulin-producing cells
}

\author{
Donghui Zhang ${ }^{1,2, *}$, Wei Jiang ${ }^{1, *}$, Meng Liu ${ }^{1,2}$, Xin Sui ${ }^{1,2}$, Xiaolei Yin ${ }^{1,2}$, Song Chen ${ }^{1}$, Yan Shi ${ }^{2}$, Hongkui Deng ${ }^{1,2}$ \\ ${ }^{1}$ Key Laboratory of Cell Proliferation and Differentiation of the Ministry of Education, College of Life Sciences, Peking University, \\ Beijing 100871, China; ${ }^{2}$ Laboratory of Chemical Genomics, Shenzhen Graduate School of Peking University, Shenzhen 518055 , \\ China
}

Human pluripotent stem cells represent a potentially unlimited source of functional pancreatic endocrine lineage cells. Here we report a highly efficient approach to induce human embryonic stem (ES) cells and induced pluripotent stem (iPS) cells to differentiate into mature insulin-producing cells in a chemical-defined culture system. The differentiated human ES cells obtained by this approach comprised nearly $25 \%$ insulin-positive cells as assayed by flow cytometry analysis, which released insulin/C-peptide in response to glucose stimuli in a manner comparable to that of adult human islets. Most of these insulin-producing cells co-expressed mature $\beta$ cell-specific markers such as NKX6-1 and PDX1, indicating a similar gene expression pattern to adult islet $\beta$ cells in vivo. In this study, we also demonstrated that EGF facilitates the expansion of PDX1-positive pancreatic progenitors. Moreover, our protocol also succeeded in efficiently inducing human iPS cells to differentiate into insulin-producing cells. Therefore, this work not only provides a new model to study the mechanism of human pancreatic specialization and maturation in vitro, but also enhances the possibility of utilizing patient-specific iPS cells for the treatment of diabetes.

Keywords: insulin-producing cell, pancreatic differentiation, human embryonic stem cells, human induced pluripotent cells Cell Research (2009) 19:429-438. doi: 10.1038/cr.2009.28; published online 3 March 2009

\section{Introduction}

Islet transplantation has been suggested to be a promising treatment for type 1 diabetes, and human embryonic stem (ES) cells are potentially an unlimited cell source of transplantable islet cells [1]. Moreover, the recently developed strategies for obtaining human induced pluripotent stem (iPS) cells have indicated the possibility of generating functional, patient-specific cell types [2-5]. Therefore, the identification of new approaches to induce human ES cells or iPS cells to differentiate into insulinproducing cells will enhance the potential for patientspecific cell transplantation therapy in diabetes.

At present, several groups, including us, have reported stepwise protocols for mimicking the mechanism of in

\footnotetext{
*These two authors contributed equally to this work. Correspondence: Hongkui Deng

Tel: +86-10-6275-6474; Fax: +86-10-6275-6954

E-mail: hongkui_deng@pku.edu.cn

Received 9 January 2009; revised 1 February 2009; accepted 4 February 2009; published online 3 March 2009
}

vivo pancreas development. These protocols induce definitive endoderm differentiation at the first stage, then pancreatic specialization and maturation at the following stages. A stepwise combination of different growth factors and small molecules was utilized to direct human ES cells to differentiate into insulin-producing cells [6-9]. In these reported protocols, insulin-positive cells were that exhibited some characteristics of pancreatic islet cells were generated. Most of these cells released insulin and C-peptide, expressed certain islet transcription factors and contained secretory granules [6-9]. However, these insulin-positive cells mostly exhibited immature islet characteristics such as the co-expression of insulin/ C-peptide and glucagon [6, 7] and low insulin/C-peptide content in the differentiated cells $[8,9]$. In general, the differentiation efficiency of the insulin-producing cells derived from human ES cells was quite low and the insulin/C-peptide-secreting level of these cells in vitro was much lower than that of adult human islet cells. Therefore, further efforts are needed to not only improve differentiation efficiency, but also promote insulin-producing cell maturation in vitro. 
Here, we describe a highly efficient strategy to direct both human ES cells and human iPS cells to differentiate into mature insulin-producing cells similar to adult islet $\beta$ cells. The differentiated human ES cells at the final stage secreted C-peptide in response to glucose stimulation in a manner approaching that of adult human islets. Furthermore, the co-expression of C-peptide, PDX1 and NKX6-1 within induced insulin-producing cells was observed in vitro for the first time. Based on this in vitro differentiation model, EGF has been further identified to be important for human pancreatic lineage cell expansion and maturation.

\section{Results}

\section{A highly efficient step-wise pancreatic differentiation strategy}

We have developed a highly efficient step-wise protocol to direct pancreatic differentiation from the human ES cell line H9 (Figure 1A). First, activin A and wortmannin were utilized to induce definitive endoderm formation. The specific marker gene expression patterns in the endoderm were monitored and the expression peaks of Sox 17 and Foxa 2 were observed on day 4 (Figure 1B), at a time when the expression level of Oct4 was rapidly reduced (Figure 1C). Moreover, the $T$ marker exhibited a peak expression at day 2 (Figure 1B), which indicated the induced endoderm was a definitive endoderm derived from mesendoderm. Furthermore, we checked the coexpression of CXCR4 and SOX17 to confirm the definitive endoderm and the flow cytometry data showed, that the culture at day 4 comprised about $>95 \%$ CXCR4and SOX17-double-positive cells (Figure 1F). Second, the differentiated endoderm cells were treated with RA,
NOGGIN and FGF7 to induce pancreatic specialization. On day 8 , the upregulated gene expression of $P d x 1$, Pax4, Pax6 and Hnf6 was detected (Figure 1D), which suggested pancreatic specialization.

After EGF-regulated progenitor expansion, we designed a cocktail of factors to induce the progenitors to mature. The expression of MafA, insulin, Glut2, Nkx6-1, Glucokinase and Tcfl emerged and reached a peak on day 20 (Figure 1E), which indicated the appearance of mature insulin-producing cells. After approximately 20 days of induction, insulin-positive cells were observed and their percentage reached approximately $25 \%$ (Figure $1 \mathrm{G})$. Compared with the previously reported efficiency of $4.1 \%$ or $7.3 \%$ for insulin/C-peptide-positive cells as assayed by flow cytometry analysis $[6,7]$, our approach resulted in higher efficiency. Moreover, the expression dynamics of most specific $\beta$ cell marker genes during insulin-producing cell differentiation from human ES cells in vitro (Figure 1D and 1E) were similar to that of pancreatic $\beta$ cell development in vivo $[10,11]$, suggesting that our protocol recapitulates key developmental stages in human pancreatic $\beta$ cell specialization. Similar results were observed in another human ES cell line, H1 (Supplementary information, Figure S1).

\section{EGF promotes the expansion of induced PDX1-positive} pancreatic progenitor cells

During stage 3 in our approach, the population of PDX1-positive cells was observed to be robust. Many PDX1-positive cells co-expressed Ki67, a mitosis marker, which indicated a population of proliferating PDX1positive cells (Figure 2A). We also observed the coexpression of PDX1, FOXA2, SOX9 and HNF1B (Figure $2 \mathrm{~B})$, which is consistent with the observation that the

Figure 1 Differentiation of human ES cells into insulin-producing cells by a new step-wise protocol. (A) The sketch of human ES cell differentiation protocol for obtaining mature insulin-producing cells. First, activin A and wortmannin were utilized to induce definitive endoderm formation. Second, the differentiated endoderm cells were treated with RA, NOGGIN and FGF7 to induce pancreatic specialization. Finally, after EGF-regulated pancreatic progenitor expansion, a cocktail of factors was utilized to induce mature insulin-producing cells. Act A, activin A; Wort, wortmannin; nico, nicotinamide; Ex-4, Exendin-4. (BE) The dynamic gene expression patterns of the differentiated human ES cells were analyzed every two days after induction from three separate experiments by quantitative RT-PCR analysis. (B) The mesendoderm marker $T$ exhibited a peak expression at day 2 and the expression peaks of definitive endoderm markers Sox17 and Foxa2 were observed on day 4 . (C) The expression level of Oct4, a human ES cell pluripotency marker, was rapidly reduced around day 2. (D) On day 8 , the upregulated gene expression of Pdx1, Pax4, Pax6 and Hnf6 was first detected, which exhibited a peak expression around day 20. (E) The pancreatic maturation markers including MafA, insulin, Glut2, Nkx6-1, Glucokinase and Tcf1 emerged and reached a peak on day 20. (F) Flow cytometry analysis of differentiated human ES cells revealed that after 4 days' induction with Act and Wort, $96.4 \%$ of cells were SOX17- and CXCR4-double-positive cells. The right panel shows the human ES cell-derived SOX17 positive cells detected by immunostaining. (G) Flow cytometry analysis revealed that the differentiated human ES cells at the final maturation stage comprised $25.3 \%$ insulin-positive cells. The right panel shows the human ES cell-derived insulin-positive cells detected by immunostaining of insulin and the inserted magnifying image shows the clear cytoplasmic location of insulin. Similar results were obtained in at least three independent experiments. Scale bar $=100 \mu \mathrm{m}$. 
A

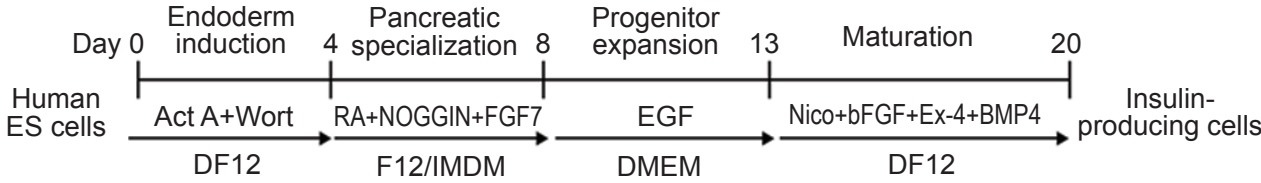

B

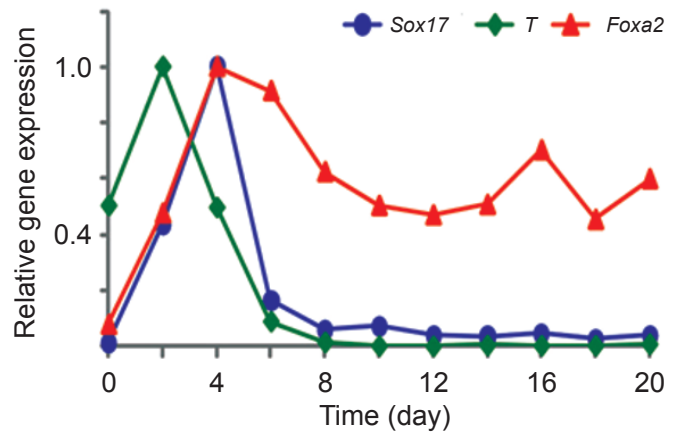

D

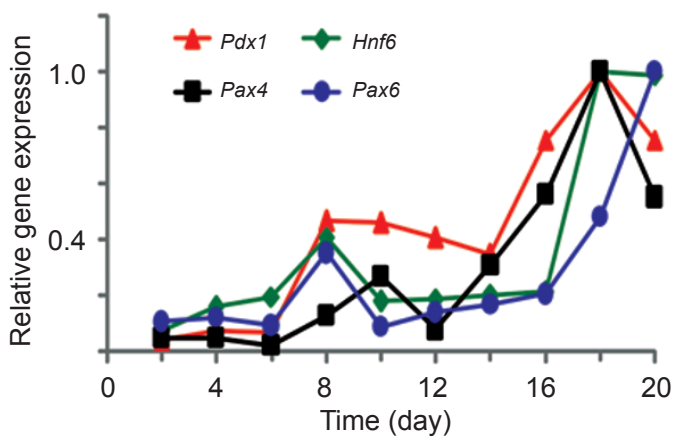

F
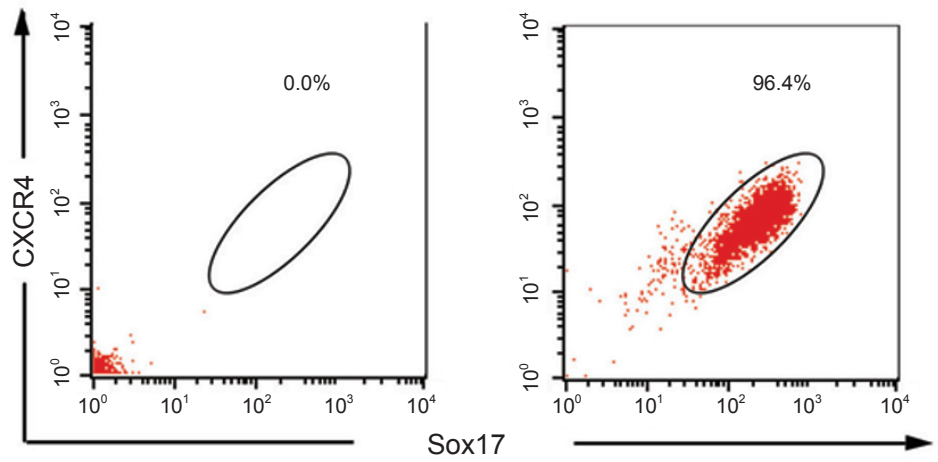

G
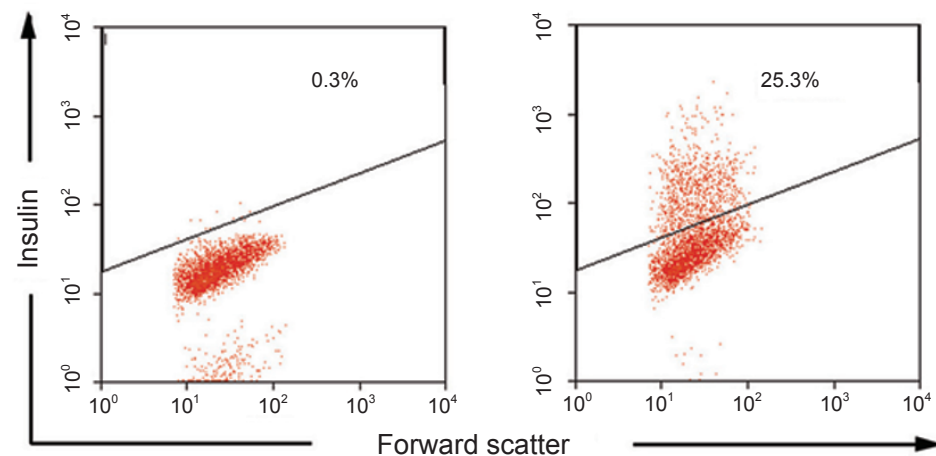

C

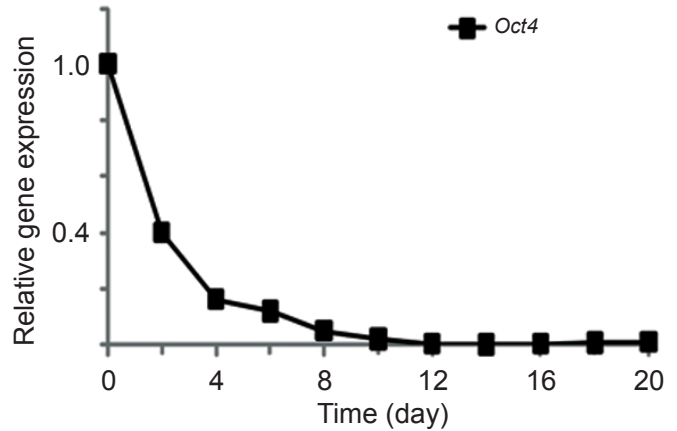

E

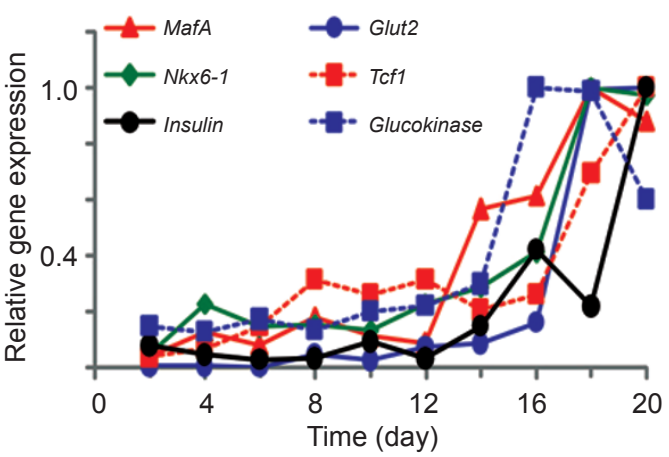

SOX17 DAPI

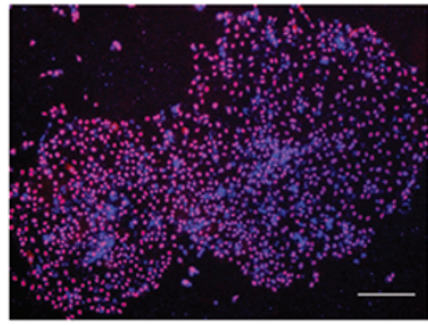

Insulin DAPI

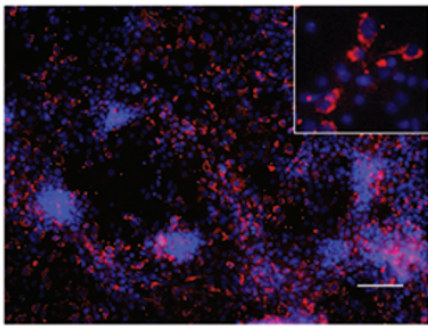


A
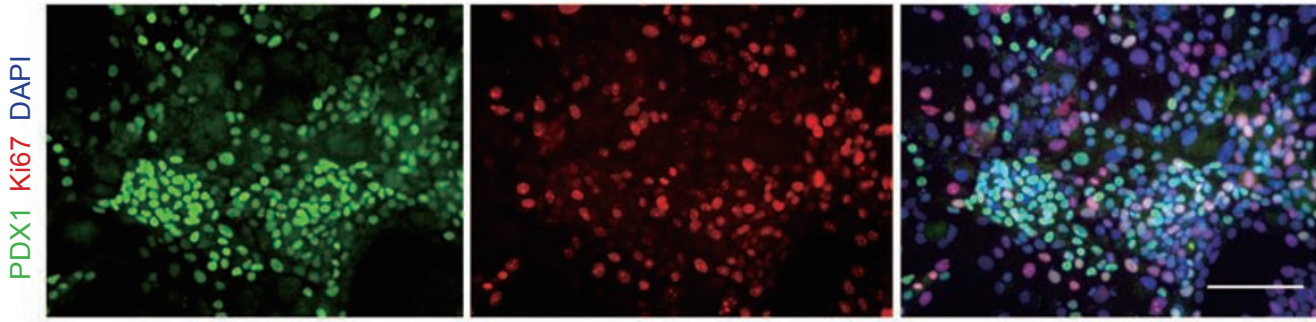

B
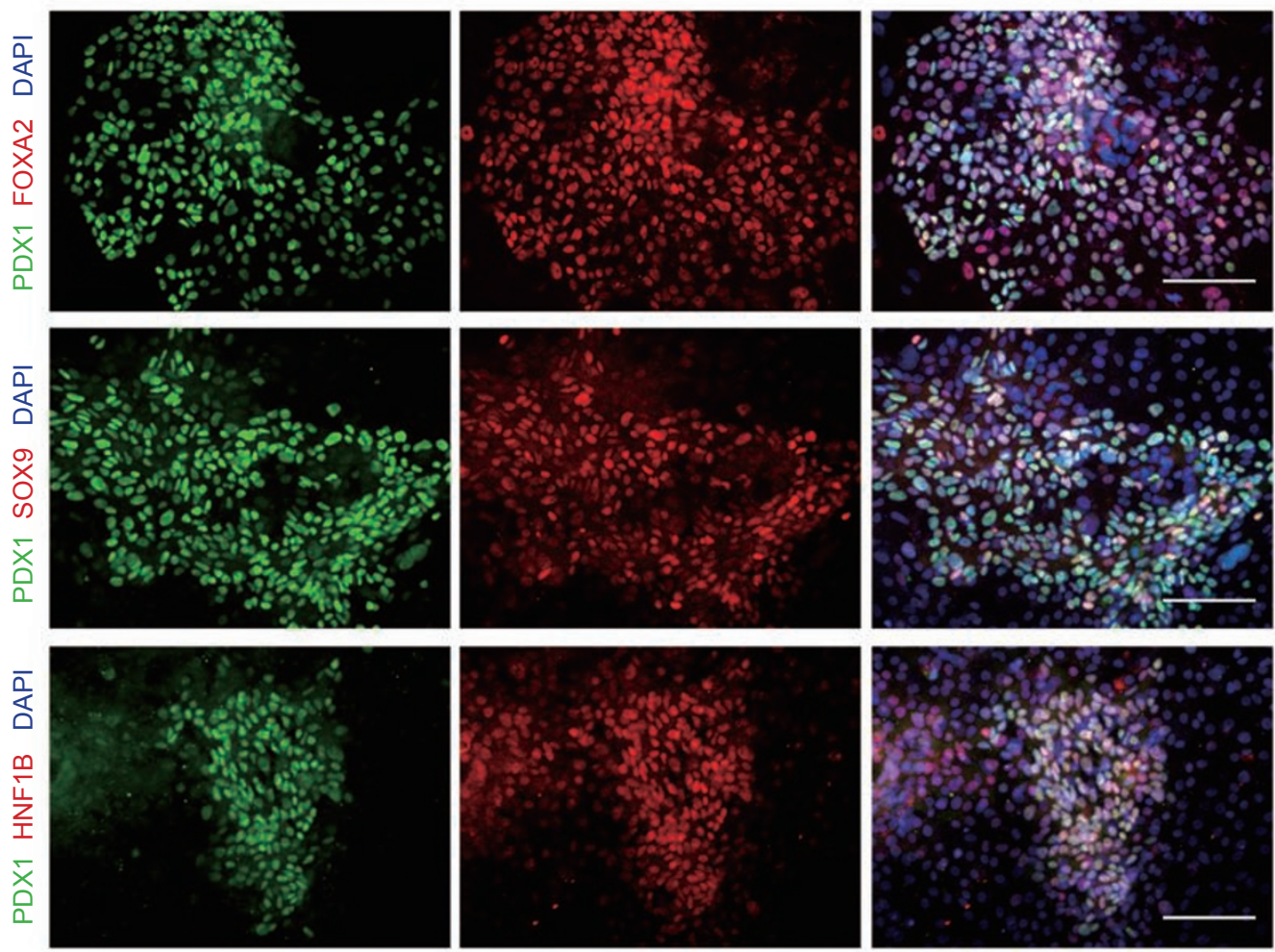

C

with EGF

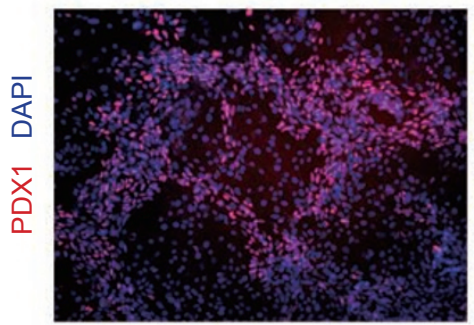

D

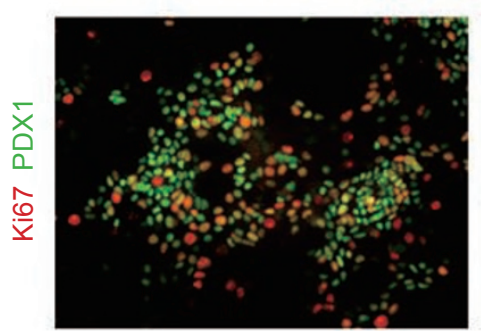

w/o EGF
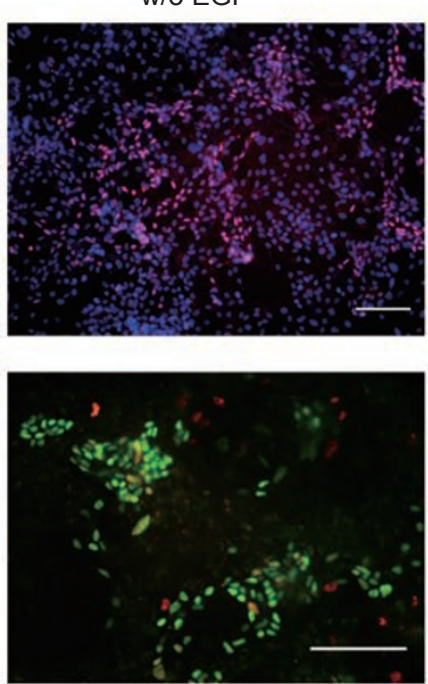
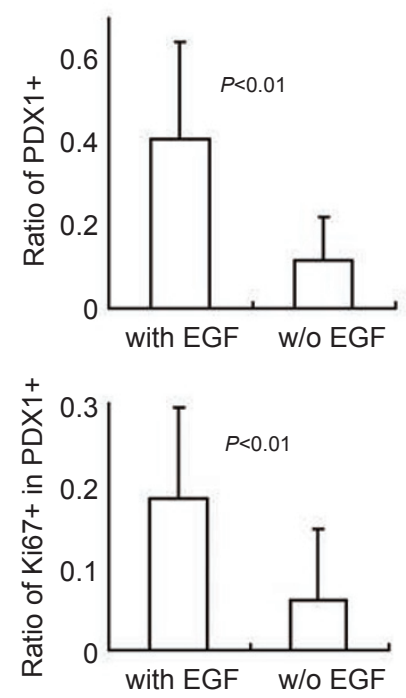
Figure 2 EGF promotes the expansion of human ES cell-derived PDX1-positive pancreatic progenitor cells. Immunoassay of differentiated human ES cells at the third induction stage with EGF treatment. (A) Many PDX1-positive cells co-expressed Ki67. (B) The co-expression of PDX1 with FOXA2, SOX9 and HNF1B, respectively, could also be detected at the progenitor expansion stage. (C) The human ES cell-derived PDX1-positive cells at the progenitor expansion stage with EGF treatment nearly two times more than the cells without EGF treatment, as detected by the immunostaining assay. The right panel shows the statistical data $(P<0.01)$. (D) Immunoassay of PDX1 and Ki67 co-expression of the induced human ES cells at the progenitor expansion stage indicated that EGF treatment also improved the proliferation of PDX1-positive cells. The right panel showed the statistical data (right, $P<0.01$ ). Scale bar $=50 \mu \mathrm{m}$.

four transcription factors were expressed in pancreatic progenitor stage in vivo $[12,13]$. These data indicate that our approach did successfully induce pancreatic progenitor differentiation from human ES cells.

In order to more efficiently expand the pancreatic progenitors, we screened several growth factors. EGF was found to be able to significantly increase the efficiency of PDX1-positice cells differentiated from induced human ES cells (Figure 2C, 38.6\% vs $12.8 \%, P<0.01$ ), which was consistent with our previous study in mouse nuclear-transfer ES cells [14]. We further evaluated the effect of EGF on proliferative PDX1-positive cells and demonstrated that EGF also improved the proliferation of PDX1-positive cells (Figure 2D, 20.1\% vs 6.8\%, $P$ $<0.01)$. These data indicated the important role of EGF signaling in pancreatic progenitor expansion.

The insulin-producing cells from human ES cells display characteristics similar to those of pancreatic $\beta$ cells

To further characterize the generated insulin-producing cells at the final stage, we examined their gene expression at both mRNA and protein levels. The expression of islet cell-specific marker genes ( $P d x 1$, Glut2, MafA, Nkx6-1, Isl-1 and NeuroD) was clearly detected by RT-PCR (Figure 3A). C-peptide co-expressed with PDX1 (Figure 3B) and we did not detect insulin/somatostatin co-expression (Figure 3C) or insulin/glucagon co-expression (data not shown) in cells, which is consistent with the hormone expression pattern of mature islets.

Furthermore, we checked the gene expression pattern of the key transcription factors for $\beta$ cells. The crucial transcription factors PDX1 and NKX6-1 were coexpressed with insulin or C-peptide (Figure 3D), which confirmed their mature pancreatic $\beta$ cell characteristics. Therefore, the specific expression pattern of these important pancreatic hormones and transcription factors strongly indicates that mature islet-like $\beta$ cells had been obtained in vitro at the final stage [11].

Based on the above findings, we further analyzed the potential of glucose-stimulated C-peptide release, which is one of the most important physiological function indices in $\beta$ cells. The $\mathrm{C}$-peptide release with $\mathrm{KCl}$ stimula- tion was significantly higher than that with low glucose (9.92 vs $2.75 \mathrm{ng} / \mathrm{mg}$ protein, $P<0.005$ ), which indicated that the differentiated cells secreted C-peptide in response to physiological $\mathrm{KCl}$ stimulation. Furthermore, C-peptide release by the differentiated insulin-producing cells was increased by high glucose treatment to approximately two-fold over basal glucose stimulation (5.55 vs $2.75 \mathrm{ng} / \mathrm{mg}$ protein, $P=0.01$ ), in a manner comparable to that of adult human islet (41.2 vs $16.1 \mathrm{ng} / \mathrm{mg}$ protein, $P<0.005$ ) (Figure 3E).

Human iPS cells could be directed to differentiate into pancreatic insulin-producing cells

We further attempted to direct human iPS cells to differentiate into insulin-producing cells using this approach. Recently, our lab has developed a highly efficient strategy to generate fully reprogrammed human iPS cells [15]. Based on this technique, we generated a panel of human iPS cells by virally transducing Oct4, Sox 2 and Klf4, while omitting $c M y c$ to decrease tumorigenicity risk [16]. The generated iPS cells expressed pluripotency marker genes such as human ES cell line H9, including SSEA4, NANOG, TRA-1-60 and TRA-1-81 (Supplementary information, Figure S2A). We transplanted these cells into immunodeficient mice and observed the effect of the transplant. Further histochemical analysis confirmed the transplant comprised three germ layers (Supplementary information, Figure S2B).

The generated human iPS cells were utilized for pancreatic differentiation. Most human iPS cell lines were induced into PDX1-positive progenitor cells (Figure 4A) and further differentiated into final stage cells expressing Pdx1, MafA, Glut2 and insulin, which were comparable to the human ES cell line H9 (Figure 4B). For a representative cell line $\mathrm{C} 1$, after first-stage induction, the expression of SOX17 was initiated (Figure 4C). Following the induction process, the differentiated iPS cells were detected to express PDX1 and SOX9 step by step (Figure $4 \mathrm{C})$. At the final stage, the expression of PDX1 and insulin was observed (Figure 4C) and we also detected several cells expressing Amylase (Figure 4C), suggesting pancreatic exocrinal cell differentiation. To further confirm 
A

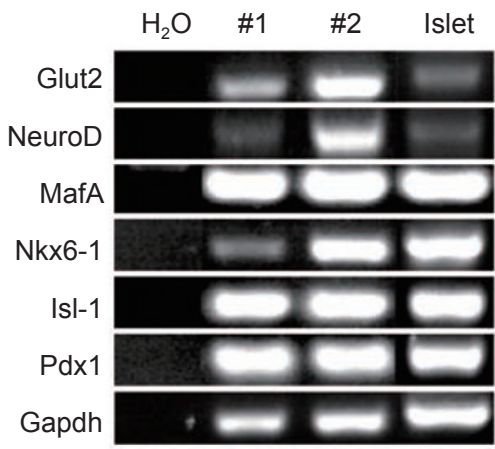

E

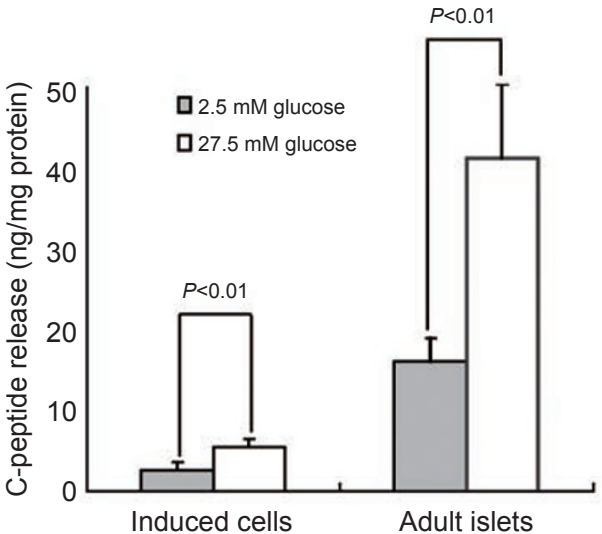

B
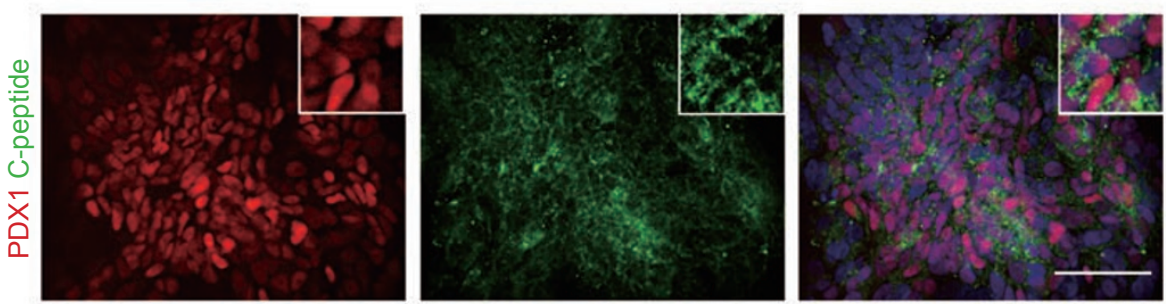

C
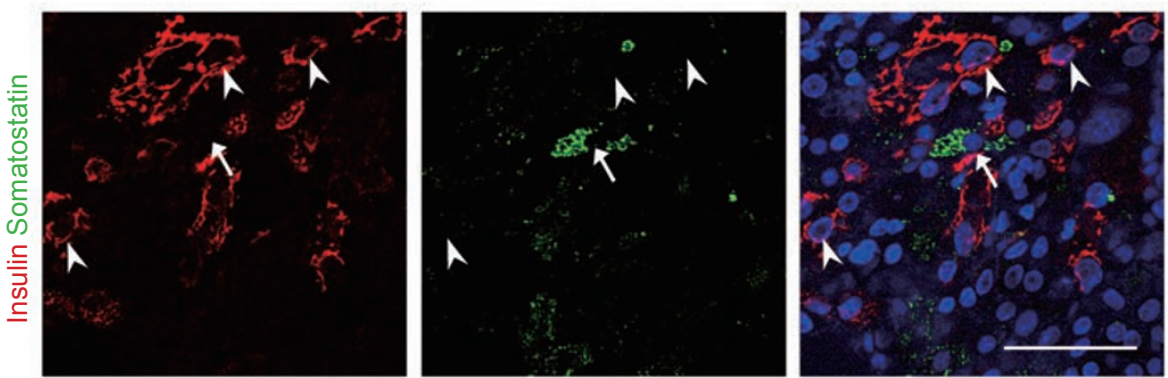

D
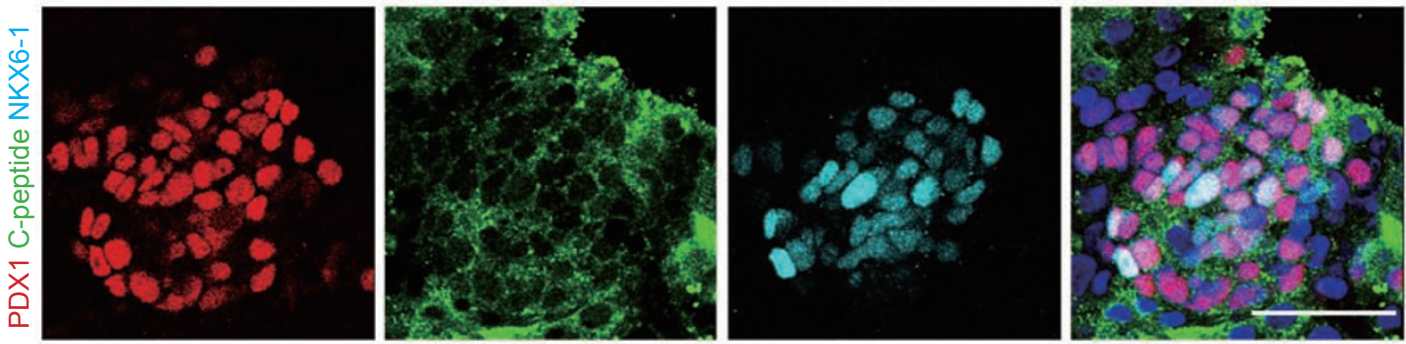

Figure 3 The pancreatic $\beta$ cell characters of the differentiated human ES cells at the final maturation stage. (A) The differentiated human ES cells at the final maturation stage expressed islet cell specific markers such as Pdx1, Glut2, MafA, Nkx6-1, Isl-1 and NeuroD by RT-PCR analysis. \#1 and \#2 represent two independent samples. Immunoassay of the differentiated cells at the final mature stage for expression of PDX1 and C-peptide (B), insulin and somatostatin (C), PDX1, C-peptide and NKX6-1 (D), which indicated that the hormone expression pattern of the differentiated human ES cells induced with our step-wise protocol is similar to that of mature islets. The right panel shows the merged figures with DAPI staining. The inserted magnifying image in (B) showed the clear cellular location of PDX1 and C-peptide. In (C), the arrow indicates the somatostatin-positive cells and the arrowhead indicates the insulin-positive cells; there were no somatostatin and insulin-double-positive cells observed. Scale bar $=50 \mu \mathrm{m}$. (E) The glucose-stimulated C-peptide release of the differentiated insulin-producing cells at the maturation stage is two-fold over that in the basal glucose condition, which is comparable to the adult human islet response with glucose stimuli. 
the insulin-producing cells, we examined the expression of C-peptide and PDX1. We observed the co-expression of PDX1 and C-peptide (Figure 4D). These results suggest that human iPS cells can be efficiently differentiated into pancreatic lineage cells by our stepwise induction protocol.

\section{Discussion}

We have established a highly efficient approach for inducing human ES cells to differentiate into mature pancreatic insulin-producing cells in vitro. This culture produced an average of $25 \%$ insulin-positive cells as assessed by flow cytometry. On the basis of this approach, we also succeeded in efficiently directing human iPS cells into insulin-producing cells. The successful approach used to produce human ES cell- and iPS cellderived insulin-producing cells opens a new avenue for the use of cell therapy in diabetes in the future.

In this study, it is demonstrated that the characteristics of the insulin-producing cells at the final stage are similar to mature islet $\beta$ cells in vivo. First, the differentiated insulin-producing cells obtained by this approach expressed most of the crucial $\beta$ cell transcription factors and functional markers, including $P d x 1$, MafA, Nkx6-1, NeuroD, Isl-1 and Glut2 (Figure 3A). Second, the coexpression of C-peptide, PDX1 and NKX6-1 (Figure 3D) was observed in the induced insulin-producing cells in vitro. The co-expression of insulin, PDX1 and other key transcription factors, such as NKX6-1 and MaFA, is considered to be a specific functional characteristic of mature $\beta$ cells [11], and has previously been reported only in islet $\beta$ cells in vivo to our knowledge. Third, these insulin-producing cells at the final stage also could secrete insulin/C-peptide in response to glucose stimulation. The C-peptide release of differentiated cells generated by our approach under glucose stimulation was at approximately $5.55 \mathrm{ng} / \mathrm{mg}$ protein, while the corresponding value for adult human islets was $41.2 \mathrm{ng} / \mathrm{mg}$ protein (Figure $3 \mathrm{E})$. Considering that the differentiated human ES cells comprised around $25 \%$ insulin-positive cells, the levels of glucose-stimulated C-peptide release between our differentiated insulin-producing cells and adult human islets were comparable. Moreover, the quantitative PCR-based gene expression profiling demonstrated that the pancreatic differentiation induced by our approach closely parallels the key gene expression pattern of in vivo pancreas development (Figure 1C and 1D). Therefore, utilizing this strategy, it was possible to successfully generate mature and functional insulin-producing cells, the characteristics of which are similar to islet $\beta$ cells in vivo. In our future work, we will transplant the differentiated insulin- producing cells into diabetic immuno-deficient mouse to further evaluate their in vivo function.

In this study, it is shown that EGF effectively expands PDX1-positive pancreatic progenitor cells (Figure 2C and 2D), suggesting an important role for EGF signaling in human pancreatic progenitor emergence and proliferation for the first time. We previously reported that EGF enhanced the expression of PDX1 from mouse ES cells [14]. An investigation of an EGF receptor knockout mouse model showed that the development of $\beta$ cells was delayed and inhibited when the EGF pathway was repressed in vivo [17], which supports our results of in vitro human ES cell differentiation. Altogether, these results suggest a conserved role of EGF signaling in human pancreatic development. In this regard, we also observed a population of cells co-expressing PDX1 with SOX9, FOXA2 and HNF1B (Figure 2B). These key transcription factors are reported to form a functional gene expression network in pancreatic progenitor cells in vivo $[12,13]$. The same gene expression pattern of human ES cell-derived intermediates generated by our differentiation approach indicates similar characteristics between human ES cell-derived pancreatic progenitors and mouse pancreatic progenitor cells in vivo. Recently, Kroon E et al. transplanted the human ES cell-derived pancreatic endoderm into immunodeficient mice and observed glucose-responsive insulin-secreting cells, suggesting that the in vitro obtained pancreatic precursors could mature in vivo [18]. However, teratoma formation was observed in a small number of cases [18], which might be related to residual pluripotent cells in transplantation. In our system, we identified a population of pancreatic progenitors by SOX9, FOXA2, HNF1B and PDX1 co-expression, which effectively proliferated upon EGF stimulation and matured into insulin-producing cells in vitro. These expandable progenitor cells could be utilized to explore the surface markers needed to purify this cell population for transplantation in future study, which could then provide another cell source for transplantation.

In summary, we established a highly efficient approach to induce human ES cells and iPS cells to differentiate into mature insulin-producing cells in a chemical-defined culture system. We also demonstrate that EGF facilitates the expansion of PDX1-positive pancreatic progenitors. Using this approach, the obtained differentiated insulinproducing cells exhibited most of the characteristics of mature $\beta$ cells, including insulin/C-peptide release in response to glucose stimuli in a manner comparable to that of adult human islets. This newly established strategy would not only provide an efficient method to utilize patient-specific iPS cells for the treatment of diabetes, but also facilitate the future study of the mechanism of 
A
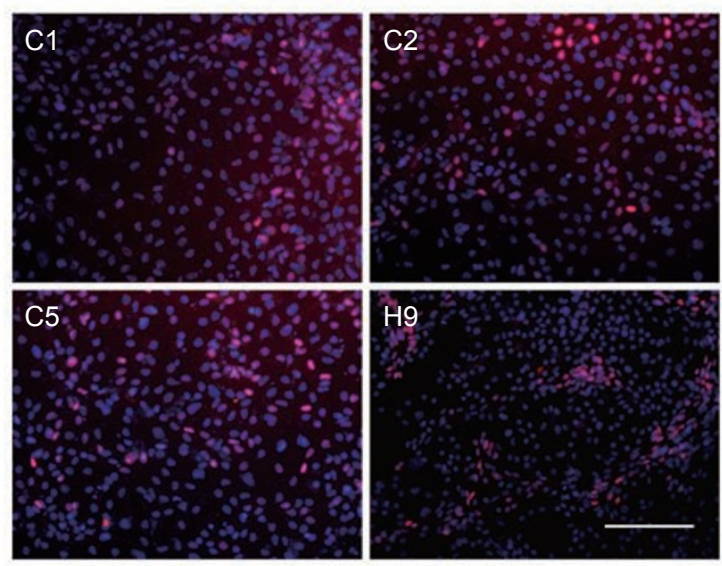

B

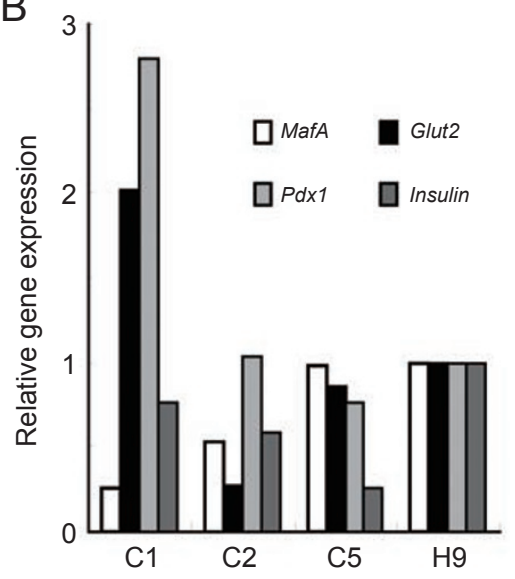

C
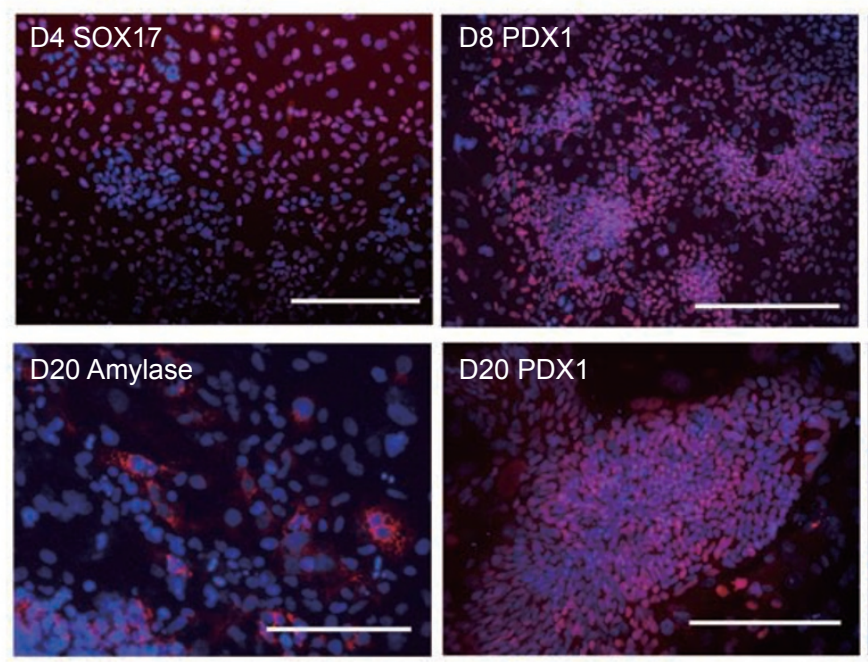

D
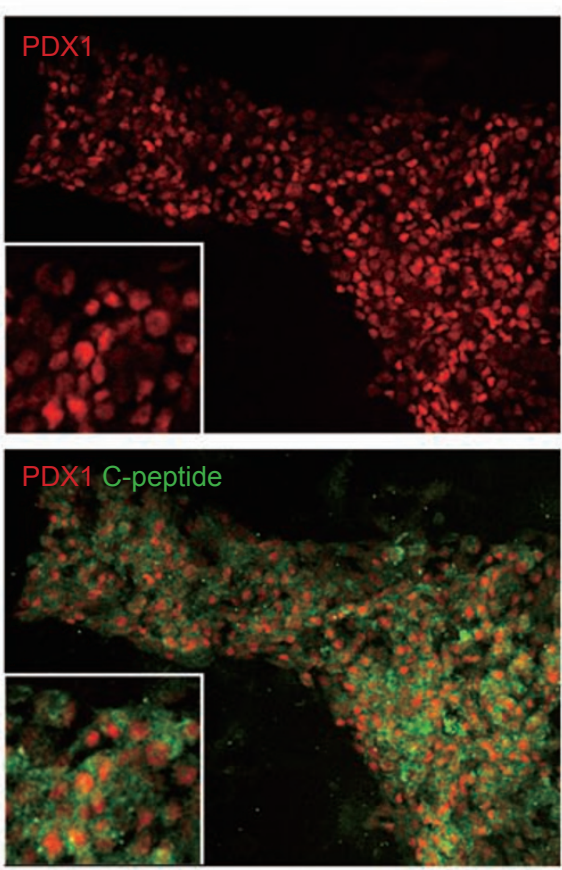
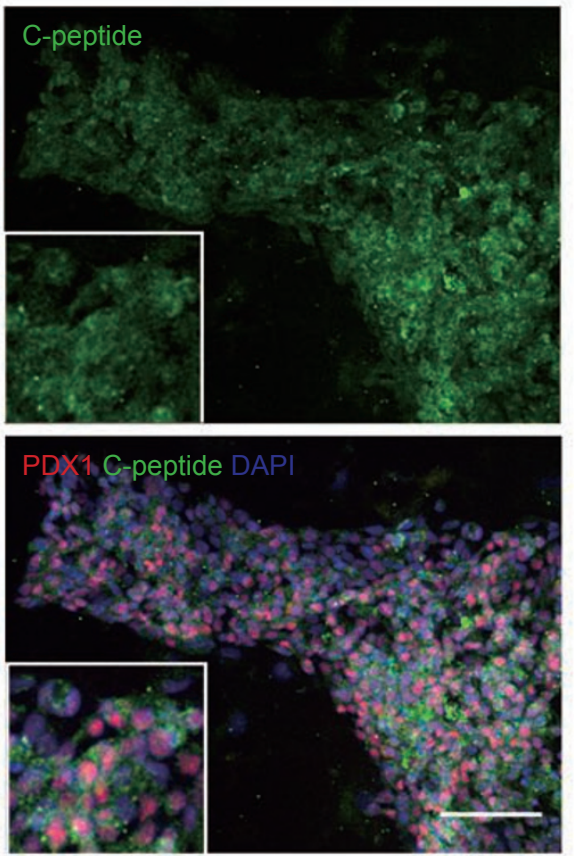
Figure 4 Differentiation of human iPS cells into pancreatic insulin-producing cells. (A) Immunostaining showing that three human iPS cell lines (named C1, C2 and C5) could all differentiate into PDX1-positive cells by our induction protocol, similar to the human ES cell line H9. (B) These three human iPS cell lines could be induced to express islet cell specific marker genes including MafA, Glut2, Pdx1 and Insulin at the final induction stage as detected by RT-PCR analysis. The relative expression value was normalized to $\mathrm{H} 9$. (C) Immunoassay showing that differentiated human iPS cells expressed marker genes at different induction stages: SOX17 on day 4, PDX1 on day 8, SOX9 on day 13, Amylase, PDX1 and Insulin on day 20. (D) Immunoassay showing that the differentiated human iPS cells at the final mature stage co-expressed PDX1 and C-peptide. The right panel showes the merged figures with DAPI staining. The inserted magnifying image showes the clear cellular location of PDX1 and C-peptide. Scale bar $=100 \mu \mathrm{m}$.

human pancreatic specialization and maturation in vitro.

\section{Materials and Methods}

\section{Culturing and differentiation of human ES cells and iPS cells into pancreatic $\beta$ cells}

Human iPS cells were generated by transducing the lentivirus containing Oct-4, Sox2 and Klf4 into human fibroblasts [15]. Human ES cells (H9 and H1) and iPS cells were cultured in DMEM/ F12 with 20\% KnockOut serum replacement (KSR) containing 10 $\mathrm{ng} / \mathrm{ml}$ of bFGF on a mitomycin-C-treated feeder layer. For differentiation, ES cells and iPS cells were dissociated into small clumps by $200 \mathrm{U} / \mathrm{ml}$ collagenase IV $\left(4 \mathrm{~min}\right.$ at $\left.37^{\circ} \mathrm{C}\right)$ and the undifferentiated colonies were collected by sedimentation. The dissociated colonies were plated into Matrigel (BD Biosciences, 1:50)-coated dishes (Falcon) for attachment with a coverage of $60 \%$ and then incubated with DMEM/F 12 (supplemented with $0.2 \%$ BSA (Sigma), $0.5 \times \mathrm{N} 2$ and $0.5 \times \mathrm{B} 27$ ) containing $100 \mathrm{ng} / \mathrm{ml}$ activin A and $1 \mu \mathrm{M}$ wortmannin for 4 days. Then the differentiated cells were cultured in F12/IMDM (1:1, supplemented with $0.5 \%$ BSA, $0.5 \%$ ITS and $0.5 \times$ B27) with $2 \mu \mathrm{M}$ RA (Sigma), $20 \mathrm{ng} / \mathrm{ml} \mathrm{FGF7}$ and $50 \mathrm{ng} / \mathrm{ml}$ NOGGIN for 4 days. Then, the cells were cultured in DMEM (high glucose; supplemented with $0.5 \%$ BSA, $1 \%$ ITS and $1 \times \mathrm{N} 2$ ) with $50 \mathrm{ng} / \mathrm{ml}$ EGF (Sigma) for 5 days and the cells exhibited obvious expansion and reached nearly $100 \%$ coverage. For maturation, another 7-9 days incubation in DF12 media with $1 \%$ ITS, $10 \mathrm{ng} / \mathrm{ml}$ bFGF, $10 \mathrm{mM}$ nicotinamide (Sigma), $50 \mathrm{ng} / \mathrm{ml}$ Exendin-4 and $10 \mathrm{ng} / \mathrm{ml} \mathrm{BMP4}$ for maturation was performed. All media and supplement were from Gibco and growth factors were from Peprotech, unless specified as otherwise.

\section{Immunofluorescence assay}

The sample cells were fixed in $4 \%$ paraformaldehyde and blocked with $10 \%$ serum and $0.2 \%$ triton X-100 in PBS, and then incubated with primary antibody overnight at $4{ }^{\circ} \mathrm{C}$ and further incubated with secondary antibody (rhodamine-labeled goat antimouse IgM, Santa Cruz; FITC or TRITC or Cy5-conjugated donkey anti-rabbit or anti-goat or anti-mouse or anti-guinea pig IgG, Jackson Lab.). The used primary antibodies are as follows: antiOCT4 (rabbit IgG, 1:200, Abcam); anti-NANOG (rabbit IgG, 1:50, R\&D Systems); anti-SSEA4 (mouse IgG, 1:40, Chemicon); antiTRA-1-60 (mouse IgM, 1:40, Chemicon); anti-TRA-1-81 (mouse IgM, 1:40, Chemicon); anti-SOX17 (goat IgG, 1:200, R\&D Systems); anti-FOXA2 (goat IgG, 1:200, R\&D Systems); anti-PDX1 (rabbit IgG, 1:1 000, Abcam; goat IgG, 1:3 000, Abcam;); antiSOX9 (rabbit IgG, 1:200, Santa Cruz); anti-HNF1B (goat IgG, 1:200, Santa Cruz); anti-Amylase (rabbit IgG, 1:500, Sigma); anti-insulin (guinea-pig IgG, 1:500, Dako); anti-C-peptide (rabbit IgG, 1:200, Linco; goat IgG, 1:200, Linco); anti-NKX6-1 (mouse $\operatorname{IgG}, 1: 100$, DSHB) and anti-Somatostatin (goat IgG, 1:200, Santa Cruz). Images were captured using an Olympus phase contrast fluorescent microscope (Olympus IX-71) or LEICA confocal microscope TCS-SP2.

\section{Flow cytometry}

Single-cell suspensions of differentiating human ES cell cultures were obtained by dissociating cells with $0.25 \%$ trypsin. Intracellular antibody staining was performed using Cytofix/Cytoperm and Perm/Wash buffer (Becton Dickinson), according to the manufacturer's instructions. The following primary and secondary antibodies were used: anti-insulin (guinea-pig IgG, 1:500, Dako), anti-CXCR4 (APC-conjugated mouse IgG, 1:200, R\&D Systems), anti-SOX17 (goat IgG, 1:200, R\&D Systems), donkey anti-mouseCy5 (1:200, Jackson Lab.), donkey anti-guinea pig-Cy3 (1:200, Jackson Lab.) and donkey anti-goat-FITC (1:200, Jackson Lab.). Flow cytometry data were acquired on a Becton Dickinson FACSCalibur and analyzed using CellQuest Software (Becton Dickinson). For each sample, at least three independent experiments were performed.

\section{RT-PCR and quantitative PCR analysis of gene expression}

Reverse-transcription PCR and quantitative PCR were performed as described previously [14]. Before reversetranscription, about $5 \mu \mathrm{g}$ of RNA was digested by RNase-free DNase I (Ambion) to remove genomic DNA contamination. The primer sequences and the length of the product are shown in Supplementary information, Table S1. Quantitative PCR analysis was performed on ABI PRISM 7300 Sequence Detection System using the SYBR Green PCR Master Mix (TOYOBO). The expression level of each gene at every checkpoint was normalized to the maximal level observed, arbitrarily set as 1 . For each sample, at least three independent experiments were performed. The primer sequences and the length of the product are shown in Supplementary information, Table S2.

\section{Insulin/C-peptide release assay by ELISA}

Primary adult human islets were isolated according to a standard procedure used for human clinical trials and provided by Dr Jinning Lou (China-Japan Friendship Hospital). The human C-peptide levels in culture supernatants were measured by Human C-peptide ELISA Kit (Linco). After pre-incubation with KrebsRinger buffer at $37^{\circ} \mathrm{C}$ for $90 \mathrm{~min}$, the differentiated cells or adult human islets were incubated with Krebs-Ringer buffer containing $2.5 \mathrm{mM}$ glucose or $27.5 \mathrm{mM}$ glucose or $30 \mathrm{mM} \mathrm{KCl}$ at $37{ }^{\circ} \mathrm{C}$ for $60 \mathrm{~min}$. Then the respective conditioned supernatant was collected 
and analyzed. The total protein content was determined with a BCA $^{\text {тм }}$ Protein Assay Kit (PIERCE).

\section{Statistical analyses}

To determine the percentage of Ki67- and PDX1-positive cells, three independent experiments were performed. We utilized DAPI staining to estimate the amount of total cells. Ki67- or PDX1-positive cells and DAPI-positive cells in the whole randomly selected microscopic fields were counted from at least 10 non-consecutive fields. The percentage of Ki67- and PDX1-positive cells was calculated by dividing the number of Ki67- or PDX1-positive cells by the number of DAPI-positive cells.

All averaged data are expressed \pm standard error of the mean unless otherwise stated. Statistical analysis was performed on the data using Microsoft Excel. For comparisons of discrete data sets, unpaired Student's $t$-tests were used. Significance levels or $P$ values are stated in figure legends.

\section{Acknowledgments}

This research was supported by the Ministry of Science and Technology (Grant 2006AA02A113), Science and Technology Plan of Beijing Municipal Government (D07050701350705), Gongjian Project of Beijing Municipal Education Commission, National Basic Research Program of China (973 Program, 2007CB947900, 2009CB941200 and 2009CB941100), National Natural Science Foundation of China (30830061) and a 111 Project to H Deng. We thank Dr Jinning Lou (China-Japan Friendship Hospital) for providing adult human islets and Yizhe Zhang for technical support on real-time PCR. We also thank Yang Zhao, Han Qin, Fangfang Zhu, Zhihua Song, Jiefang You, Yuhua Han and other colleagues in our laboratory for providing technical assistance during the experiments.

\section{References}

1 Stanley EG, Elefanty AG. Building better beta cells. Cell Stem Cell 2008; 2:300-301.

2 Takahashi K, Tanabe K, Ohnuki M, et al. Induction of pluripotent stem cells from adult human fibroblasts by defined factors. Cell 2007; 131:861-872.

3 Yu J, Vodyanik MA, Smuga-Otto K, et al. Induced pluripotent stem cell lines derived from human somatic cells. Science
2007; 318:1917-1920.

4 Park IH, Arora N, Huo H, et al. Disease-specific induced pluripotent stem cells. Cell 2008; 134:877-886.

5 Dimos JT, Rodolfa KT, Niakan KK, et al. Induced pluripotent stem cells generated from patients with ALS can be differentiated into motor neurons. Science 2008; 321:1218-1221.

6 D'Amour KA, Bang AG, Eliazer S, et al. Production of pancreatic hormone-expressing endocrine cells from human embryonic stem cells. Nat Biotechnol 2006; 24:1392-1401.

7 Jiang J, Au M, Lu K, et al. Generation of insulin-producing islet-like clusters from human embryonic stem cells. Stem Cells 2007; 25:1940-1953.

8 Jiang W, Shi Y, Zhao D, et al. In vitro derivation of functional insulin-producing cells from human embryonic stem cells. Cell Res 2007; 17:333-344.

9 Phillips BW, Hentze H, Rust WL, et al. Directed differentiation of human embryonic stem cells into the pancreatic endocrine lineage. Stem Cells Dev 2007; 16:561-578.

10 Oliver-Krasinski JM, Stoffers DA. On the origin of the beta cell. Genes Dev 2008; 22:1998-2021.

11 Murtaugh LC. Pancreas and beta-cell development: from the actual to the possible. Development 2007; 134:427-438.

12 Seymour PA, Freude KK, Tran MN, et al. SOX9 is required for maintenance of the pancreatic progenitor cell pool. Proc Natl Acad Sci USA 2007; 104:1865-1870.

13 Lynn FC, Smith SB, Wilson ME, et al. Sox9 coordinates a transcriptional network in pancreatic progenitor cells. Proc Natl Acad Sci USA 2007; 104:10500-10505.

14 Jiang W, Bai Z, Zhang D, et al. Differentiation of mouse nuclear transfer embryonic stem cells into functional pancreatic beta cells. Diabetologia 2008; 51:1671-1679.

15 Zhao Y, Yin X, Qin H, et al. Two supporting factors greatly improve the efficiency of human iPS cell generation. Cell Stem Cell 2008; 3:475-479.

16 Nakagawa M, Koyanagi M, Tanabe K, et al. Generation of induced pluripotent stem cells without Myc from mouse and human fibroblasts. Nat Biotechnol 2008; 26:101-106.

17 Miettinen PJ, Huotari M, Koivisto T, et al. Impaired migration and delayed differentiation of pancreatic islet cells in mice lacking EGF-receptors. Development 2000; 127:2617-2627.

18 Kroon E, Martinson LA, Kadoya K, et al. Pancreatic endoderm derived from human embryonic stem cells generates glucose-responsive insulin-secreting cells in vivo. Nat Biotechnol 2008; 26:443-452.

(Supplementary information is linked to the online version of the paper on the Cell Research website.) 